\title{
Exact black hole solution with a minimally coupled scalar field
}

\author{
Cristián Martínez, Ricardo Troncoso and Jorge Zanelli* \\ Centro de Estudios Científicos (CECS), Casilla 1469, Valdivia, Chile.
}

\begin{abstract}
An exact four-dimensional black hole solution of gravity with a minimally coupled self-interacting scalar field is reported. The event horizon is a surface of negative constant curvature enclosing the curvature singularity at the origin, and the scalar field is regular everywhere outside the origin. This solution is an asymptotically locally AdS spacetime. The strong energy condition is satisfied on and outside the event horizon. The thermodynamical analysis shows the existence of a critical temperature, below which a black hole in vacuum undergoes a spontaneous dressing up with a nontrivial scalar field in a process reminiscent of ferromagnetism.
\end{abstract}

\footnotetext{
${ }^{*}$ E-mail: martinez@cecs.cl, ratron@cecs.cl, jz@cecs.cl
} 


\section{Introduction}

In four dimensions, exact black hole solutions in General Relativity with a minimally coupled self-interacting scalar field have not been reported previously. This fact may be seen as a natural consequence of the so called no-hair conjecture, which originally stated that a black hole should be characterized only in terms of its mass, angular momentum and electric charge [1, 2] (for recent discussions see e.g., [3]). This obstacle can be circumvented introducing a cosmological constant $\Lambda$ and a conformal coupling, in which case exact black hole solutions are known in three [四, 5] and four dimensions [6]. For vanishing $\Lambda$, a four-dimensional black hole is also known, but the scalar field diverges at the horizon [7].

However, for minimal coupling, an exact black hole solution is known only in three dimensions [5], provided $\Lambda<0$, in which case, spherically symmetric black hole solutions have also been found numerically in four [8, 9] and five dimensions [10]. On the other hand, a negative cosmological also allows for the existence of black holes whose horizon has nontrivial topology in four [11, 12, 13] and higher dimensions [14, 15] as well as for gravity theories containing higher powers of the curvature [16, 17, 18, 19].

In this paper we report an exact black hole solution in four-dimensions for gravity with a negative cosmological constant, with a minimally coupled self-interacting scalar field. The event horizon is a surface of negative constant curvature enclosing the curvature singularity at the origin. The spacetime is asymptotically locally anti-de Sitter (AdS), and the scalar field is regular everywhere outside the origin. It is shown that there is a second order phase transition at a critical temperature $T_{c}=(2 \pi l)^{-1}$, below which a black hole in vacuum undergoes a spontaneous dressing up with a nontrivial scalar field. As is shown in the appendix, the transformation of this theory to the conformal frame maps this solution into another black hole with a rich causal structure.

\section{Black hole solution}

Consider four-dimensional gravity with negative cosmological constant $\left(\Lambda=-3 l^{-2}\right)$ and a scalar field described by the action

$$
I\left[g_{\mu \nu}, \phi\right]=\int d^{4} x \sqrt{-g}\left[\frac{R+6 l^{-2}}{16 \pi G}-\frac{1}{2} g^{\mu \nu} \partial_{\mu} \phi \partial_{\nu} \phi-V(\phi)\right],
$$

where $l$ is AdS radius, and $G$ is Newton's constant. We take the following self-interaction potential

$$
V(\phi)=-\frac{3}{4 \pi G l^{2}} \sinh ^{2} \sqrt{\frac{4 \pi G}{3}} \phi
$$

which has a global maximum at $\phi=0$, and has a mass term given by $m^{2}=\left.V^{\prime \prime}\right|_{\phi=0}=-2 l^{-2}$. This mass satisfies the Breitenlohner-Friedman bound that ensures the perturbative stability of AdS spacetime [20, 21]. This potential has a natural interpretation in the conformal frame 
(see the Appendix). The field equations are

$$
\begin{aligned}
G_{\mu \nu}-\frac{3}{l^{2}} g_{\mu \nu} & =8 \pi G T_{\mu \nu}, \\
\square \phi-\frac{d V}{d \phi} & =0,
\end{aligned}
$$

where $\square \equiv g^{\mu \nu} \nabla_{\mu} \nabla_{\nu}$, and the stress-energy tensor is given by

$$
T_{\mu \nu}=\partial_{\mu} \phi \partial_{\nu} \phi-\frac{1}{2} g_{\mu \nu} g^{\alpha \beta} \partial_{\alpha} \phi \partial_{\beta} \phi-g_{\mu \nu} V(\phi) \text {. }
$$

A static black hole solution with topology $\mathbb{R}^{2} \times \Sigma$, where $\Sigma$ is a two-dimensional manifold of negative constant curvature, is given by

$$
d s^{2}=\frac{r(r+2 G \mu)}{(r+G \mu)^{2}}\left[-\left(\frac{r^{2}}{l^{2}}-\left(1+\frac{G \mu}{r}\right)^{2}\right) d t^{2}+\left(\frac{r^{2}}{l^{2}}-\left(1+\frac{G \mu}{r}\right)^{2}\right)^{-1} d r^{2}+r^{2} d \sigma^{2}\right],
$$

and the scalar field is

$$
\phi=\sqrt{\frac{3}{4 \pi G}} \operatorname{Arctanh} \frac{G \mu}{r+G \mu} .
$$

Here $d \sigma^{2}$ is the line element of the base manifold $\Sigma$, which has negative constant curvature (rescaled to -1 ) and hence is locally isometric to the hyperbolic manifold $H^{2}$. This means that $\Sigma$ must be of the form $\Sigma=H^{2} / \Gamma$, where $\Gamma$ is a freely acting discrete subgroup of $O(2,1)$ (i.e., without fixed points). The configurations (2.6) are asymptotically locally AdS spacetimes, with a single timelike Killing vector $\partial_{t}$, provided $\Sigma$ is assumed to be compact without boundary ${ }^{1}$. As it is shown in section 3 , the mass of this solution is given by

$$
M=\frac{\sigma}{4 \pi} \mu,
$$

where $\sigma$ denotes the area of $\Sigma$. The only singularities of the curvature and the scalar field occur where the conformal factor in (2.6) vanishes, that is at $r=0$ and at $r=-2 G \mu$. The range of $r$ is taken as $r>-2 G \mu$ for negative mass, and $r>0$ otherwise. These singularities are surrounded by an event horizon located at

$$
r_{+}=\frac{l}{2}(1+\sqrt{1+4 G \mu / l}),
$$

provided the mass is bounded from below by

$$
\mu>-\frac{l}{4 G}
$$

\footnotetext{
${ }^{1}$ For $\mu=0$, these spacetimes admit Killing spinors provided $\Sigma$ is a noncompact surface 22]. Such a configuration describes the supersymmetric ground state of a warped black string and is therefore expected to be stable. As it was shown in Ref. [23], these configurations are also stable under gravitational perturbations.
} 
The causal structure is the same as for the Schwarzschild-AdS black hole, but at each point of the Penrose diagram the sphere is replaced by $\Sigma$.

For non-negative masses, the horizon radius satisfies $r_{+} \geq l$. For the massless case, the metric takes a simple form

$$
d \bar{s}^{2}=-\left(\frac{r^{2}}{l^{2}}-1\right) d t^{2}+\left(\frac{r^{2}}{l^{2}}-1\right)^{-1} d r^{2}+r^{2} d \sigma^{2}
$$

which is a locally AdS spacetime (i.e. it has constant curvature), and the scalar field vanishes. For $-l(4 G)^{-1}<\mu<0$, the horizon radius is in the range $l / 2<r_{+}<l$, and for $\mu=-l(4 G)^{-1}$, $r_{+}=l / 2$ becomes a double root which coincides with the singularities.

Note that the scalar field cannot be switched off keeping the mass fixed. In fact, there is only one integration constant $(\mu)$, and for $\phi \rightarrow 0$ the geometry approaches the massless black hole (2.11). This means that for a given mass there are two branches of different black hole solutions, the one with non-trivial scalar field (2.6, 2.7), and the vacuum solution (with $\phi=0$ ) found in Refs. [12, 13], whose metric reads

$$
d s_{0}^{2}=-\left[\frac{\rho^{2}}{l^{2}}-1-\frac{2 G \mu_{0}}{\rho}\right] d t^{2}+\left[\frac{\rho^{2}}{l^{2}}-1-\frac{2 G \mu_{0}}{\rho}\right]^{-1} d \rho^{2}+\rho^{2} d \sigma^{2},
$$

with mass $M=\sigma \mu_{0} / 4 \pi$.

\section{Thermodynamics}

The partition function for a thermodynamical ensemble is identified with the Euclidean path integral in the saddle point approximation around the Euclidean continuation of the classical solution [24]. Thus, we consider the Euclidean continuation of the action (2.1) in Hamiltonian form

$$
I=\int\left[\pi^{i j} \dot{g}_{i j}+p \dot{\phi}-N \mathcal{H}-N^{i} \mathcal{H}_{i}\right] d^{3} x d t+B
$$

where $B$ is a surface term. We consider the minisuperspace of static Euclidean metrics

$$
d s^{2}=N(r)^{2} f(r)^{2} d t^{2}+f(r)^{-2} d r^{2}+R(r)^{2} d \sigma^{2}
$$

with $0 \leq t \leq \beta$ periodic, $r \geq r_{+}$and a scalar field of the form $\phi=\phi(r)$. The inverse of the period $\beta$ corresponds to the temperature $T$, and the reduced Hamiltonian action is

$$
I=-\beta \sigma \int_{r_{+}}^{\infty} N(r) \mathcal{H}(r) d r+B
$$

where $\sigma$ is the area of the base manifold $\Sigma$, and

$$
\begin{aligned}
\mathcal{H}= & N R^{2}\left[\frac{1}{8 \pi G}\left(\frac{\left(f^{2}\right)^{\prime} R^{\prime}}{R}+\frac{2 f^{2} R^{\prime \prime}}{R}+\frac{1}{R^{2}}\left(1+f^{2}\right)\right)\right. \\
& \left.+\Lambda+\frac{1}{2} f^{2}\left(\phi^{\prime}\right)^{2}+V(\phi)\right] .
\end{aligned}
$$


The Euclidean black hole solution is static and satisfies the constraint $\mathcal{H}=0$. Therefore, the value of the action on the classical solution is just given by the boundary term $B$. This boundary term must be such that the action (3.1) attains an extremum within the class of fields considered here [25]. We now turn to the evaluation of the Euclidean action on shell. The condition that the geometries allowed in the variation should contain no conical singularities at the horizon requires

$$
\left.\beta\left(N(r)\left(f^{2}(r)\right)^{\prime}\right)\right|_{r=r_{+}}=4 \pi,
$$

which for the solution (2.6), directly yields the period $\beta$ as a function of $r_{+}$,

$$
T=\beta^{-1}=\frac{1}{2 \pi l}\left(\frac{2 r_{+}}{l}-1\right) .
$$

In what follows, we work in the canonical ensemble, that is, we consider variations of the action with constant $\beta$. The variation of the boundary term is

$$
\delta B \equiv \delta B_{\phi}+\delta B_{G}
$$

where

$$
\delta B_{G}=\frac{\beta \sigma}{8 \pi G}\left[N\left(R R^{\prime} \delta f^{2}-\left(f^{2}\right)^{\prime} R \delta R\right)+2 f^{2} R\left(N \delta R^{\prime}-N^{\prime} \delta R\right)\right]_{r_{+}}^{\infty},
$$

and the contribution from the matter sector is

$$
\delta B_{\phi}=\left.\beta \sigma N R^{2} f^{2} \phi^{\prime} \delta \phi\right|_{r_{+}} ^{\infty} .
$$

For the black hole solution $(2.6,2.7)$ the variation of the fields at infinity are

$$
\begin{aligned}
\left.\delta f^{2}\right|_{\infty} & =\frac{2 G}{l^{2}}\left(G \mu-\frac{l^{2}+3 G^{2} \mu^{2}}{r}-\frac{2 G \mu l^{2}-8 G^{3} \mu^{3}}{r^{2}}+O\left(r^{-3}\right)\right) \delta \mu, \\
\left.\delta \phi\right|_{\infty} & =\sqrt{\frac{3 G}{4 \pi}}\left(\frac{1}{r}-\frac{2 G \mu}{r^{2}}+O\left(r^{-3}\right)\right) \delta \mu, \\
\left.\delta R\right|_{\infty} & =\left(-\frac{G^{2} \mu}{r}+\frac{3 G^{3} \mu^{2}}{r^{2}}+O\left(r^{-3}\right)\right) \delta \mu
\end{aligned}
$$

and thus, we obtain

$$
\left.\delta B_{G}\right|_{\infty}=\frac{3 \beta \sigma}{4 \pi l^{2}}\left(G \mu r-4(G \mu)^{2}-l^{2} / 3+O\left(r^{-1}\right)\right) \delta \mu .
$$

Note that $\left.\delta B_{G}\right|_{\infty}$ has a potentially dangerous divergent term. As is shown in Sec. 5, this is a consequence of the slower fall-off of our metric compared with that of pure gravity with a standard localized distribution of matter [26]. This occurs because the scalar field goes to zero at infinity at a slower rate than under the usual assumptions. As a result of this there is a non-vanishing $\left.\delta B_{\phi}\right|_{\infty}$ given by 


$$
\left.\delta B_{\phi}\right|_{\infty}=-\frac{3 \beta \sigma}{4 \pi l^{2}}\left(G \mu r-4(G \mu)^{2}+O\left(r^{-1}\right)\right) \delta \mu,
$$

which exactly cancels the divergence coming from $\left.\delta B_{G}\right|_{\infty}$ and gives a finite contribution, yielding a finite expression for the total variation of the boundary term at infinity,

$$
\left.B\right|_{\infty}=-\frac{\beta \sigma}{4 \pi} \mu
$$

This is a generic effect observed for scalar fields with a self-interacting potential unbounded form below in asymptotically AdS spacetimes [5, 27, 28, 10].

The variation of the boundary term at the horizon, is obtained using

$$
\begin{aligned}
\left.\delta R\right|_{r_{+}} & =\delta R\left(r_{+}\right)-\left.R^{\prime}\right|_{r_{+}} \delta r_{+}, \\
\left.\delta f^{2}\right|_{r_{+}} & =-\left.\left(f^{2}\right)^{\prime}\right|_{r_{+}} \delta r_{+},
\end{aligned}
$$

together with (3.4), in Eqs. (3.6, 3.7). Note that $\left.\delta B_{\phi}\right|_{r_{+}}$vanishes, and hence

$$
\begin{aligned}
\left.\delta B\right|_{r_{+}} & =-\left.\frac{\beta \sigma}{16 \pi G} N\left(r_{+}\right)\left(f^{2}\right)^{\prime}\right|_{r_{+}} \delta R^{2}\left(r_{+}\right) \\
& =-\frac{\sigma}{4 G} \delta R^{2}\left(r_{+}\right) .
\end{aligned}
$$

Therefore, the boundary term at the horizon is

$$
\left.B\right|_{r_{+}}=-\frac{\sigma}{4 G} R^{2}\left(r_{+}\right) .
$$

Combining Eqs. (3.13) and (3.14), the on-shell value of the Euclidean action is found to be

$$
I=-\frac{\beta \sigma}{4 \pi} \mu+\frac{\sigma}{4 G} R\left(r_{+}\right)^{2},
$$

up to an arbitrary additive constant. Since the Euclidean action is related to the free energy (in units where $\hbar=k_{B}=1$ ) as $I=-\beta F$, then

$$
I=S-\beta M,
$$

where $M$ and $S$ denote mass and entropy, respectively.

Comparing expressions (3.15) and (3.16), the mass and entropy are identified as

$$
M=\frac{\sigma}{4 \pi} \mu,
$$

and

$$
S=\frac{\sigma}{4 G} R^{2}\left(r_{+}\right)=\frac{\text { Horizon Area }}{4 G},
$$

respectively ${ }^{2}$. With these expressions it is simple to check that the first law of thermodynamics $d M=T d S$ is satisfied.

\footnotetext{
${ }^{2}$ The mass could also be found using covariant methods, see e.g., [29].
} 


\section{Phase transition}

The specific heat can be found using Eqs. (2.9) and (3.5)

$$
C=\frac{\partial M}{\partial T}=\frac{\sigma l^{2}}{4 G}\left(\frac{2 r_{+}}{l}-1\right)
$$

which is always positive for $r_{+}>l / 2$ (i.e. $G \mu>-l / 4$ ), and therefore the black hole dressed with the scalar field can always reach thermal equilibrium with a heat bath. However, note that for a fixed temperature, the action principle (2.1), with the same boundary conditions, also admits the vacuum solution (2.12) with $\phi \equiv 0$. This raises the question of whether one black hole can decay into the other. Since $Z=\exp (-\beta F)$, this can be examined evaluating the difference between their respective free energies. The temperature of the vacuum black hole is

$$
T_{0}=\frac{1}{4 \pi}\left(\frac{3 \rho_{+}}{l^{2}}-\frac{1}{\rho_{+}}\right)
$$

where $\rho_{+} \geq l / \sqrt{3}$. Matching this with the temperature in Eq. 3.5) fixes the relation between their respective horizon radii as

$$
r_{+}=\frac{3 \rho_{+}}{4}-\frac{l^{2}}{4 \rho_{+}}+\frac{l}{2}
$$

The Euclidean action evaluated on the vacuum black hole (2.12) reads

$$
I_{0}=\frac{\sigma}{4 G} \frac{\rho_{+}^{4}+l^{2} \rho_{+}^{2}}{3 \rho_{+}^{2}-l^{2}},
$$

and hence, using Eq. (3.15), the difference between both Euclidean actions is given by

$$
\Delta I=I_{\phi}-I_{0}=\frac{\sigma}{16 G} \frac{\left(l-\rho_{+}\right)^{3}}{\rho_{+}} \frac{l^{2}+3 l \rho_{+}+4 \rho_{+}^{2}}{3 \rho_{+}^{2}-l^{2}} .
$$

Analogously, for a fixed temperature, the difference between both black hole masses is

$$
\Delta M=M_{\phi}-M_{0}=-\frac{\sigma}{8 \pi G} \frac{\left(\rho_{+}+l\right)\left(\rho_{+}-l\right)^{2}}{\rho_{+}^{2} l^{2}}\left[\rho_{+}^{2}-\frac{l \rho_{+}}{8}-\frac{l^{2}}{8}\right],
$$

which cannot be positive for the allowed range of $\rho_{+}$, i.e., $\Delta M=M_{\phi}-M_{0} \leq 0$. Similarly, since $S_{0}=\sigma \rho_{+}^{2}(4 G)^{-1}$, the entropies are found to obey $\Delta S=S_{\phi}-S_{0} \leq 0$. Both inequalities are saturated for $r_{+}=\rho_{+}=l$. However, at this radius, $\Delta I$ changes of sign, signaling the existence of a phase transition at the critical temperature

$$
T_{c}=\frac{1}{2 \pi l} .
$$

At the transition temperature, both black hole branches intersect at the massless configuration (2.11) (with $M_{\phi}=M_{0}=0$ ), describing a spacetime of negative constant curvature (i.e., locally AdS). The two phases at each side of the critical point are: 
$\mathbf{T}>\mathbf{T}_{\mathbf{c}}:$

In this phase, $\rho_{+}>l$, and both black holes have positive mass. As $\Delta I$ in Eq. (4.3) is negative, there is a greater probability for the decay of the black hole dressed with the scalar field into the bare black hole, induced by thermal fluctuations. In the decay process, the scalar black hole absorbs energy from the thermal bath, increasing its horizon radius an consequently its entropy. This suggests that in this process the scalar field is, in some sense, absorbed by the black hole.

$\mathbf{T}<\mathbf{T}_{\mathbf{c}}:$

In this phase, $\rho_{+}<l$, both black holes have negative mass, but now $\Delta I>0$, which means that the configuration with nonzero scalar field has greater probability. As a consequence, below the critical temperature, the bare black hole undergoes a spontaneous "dressing up" with the scalar field. In the process, the mass and entropy of the black hole decrease, so that the difference in energy and entropy are transferred to the heat bath.

At the critical temperature, the thermodynamic functions of the two phases match continuously, hence, the phase transition is of second order. The order parameter that characterizes the transition can be defined in terms of the value of the scalar field at the horizon,

$$
\begin{aligned}
\lambda & =\left|\tanh \sqrt{\frac{4 \pi G}{3}} \phi\left(r_{+}\right)\right| \\
& = \begin{cases}\frac{T_{c}-T}{T_{c}+T}: T<T_{c} \\
0 & : T>T_{c}\end{cases}
\end{aligned}
$$

\section{Summary and discussion}

We have shown the existence of an exact four-dimensional black hole solution of gravity with a minimally coupled self-interacting scalar field. The spacetime is asymptotically locally AdS, and has a curvature singularity hidden by an event horizon which is a surface of negative constant curvature. The scalar field is regular everywhere outside the origin.

The specific heat is positive for the entire mass range, so that thermal equilibrium with a heat bath can always be attained. Furthermore, there is a critical temperature $T_{c}=(2 \pi l)^{-1}$. For temperatures above $T_{c}$, a black hole dressed with the scalar field is likely to decay into the bare black hole (bare phase). For temperatures below $T_{c}$ however, a bare black hole would spontaneously acquire a nontrivial scalar field (scalar phase), in a phenomenon reminiscent of ferromagnetism, where the scalar field plays the role of the magnetization. It would be interesting to see how this phase transition manifests itself in the dual CFT [30].

The black hole mass is bounded by $M \geq-\sigma l(16 \pi G)^{-1}$, and it is simple to verify that the strong energy condition is satisfied for $r \geq r_{+}$, as it occurs for a Schwarzschild-AdS black hole.

The self-interacting potential $V(\phi)$ is negative and unbounded from below, possessing a global maximum at $\phi=0$. The mass term for scalar perturbations satisfies the Breitenlohner- 
Friedman bound that guarantees the perturbative stability of global AdS spacetime [20]. For the topology considered here, it was shown that the stability of the locally AdS spacetime (2.11) under scalar perturbations, holds provided the mass satisfies the same BF bound [31. However, in general, the stability is no longer guaranteed for non-perturbative solutions. For the dressed black hole considered here, the scalar field cannot be treated as a probe. This is because, even for small mass, there is a strong back reaction that reaches the asymptotic region.

The presence of matter fields with nontrivial asymptotic behavior has generically two effects: It gives rise to a back reaction that modifies the asymptotic form of the geometry, and it generates additional contributions to the charges that depend explicitly on the matter fields at infinity which are not already present in the gravitational part. These two effects have been observed in similar setups and for various dimensions in Refs. [5, 10, 27, 28, 32]. Indeed, the scalar field would not contribute to the conserved charges if it falls off as $\phi \sim r^{-3 / 2+\varepsilon}$, whereas here the asymptotic behavior of the scalar field (2.7) is

$$
\phi=\sqrt{\frac{3 G}{4 \pi}}\left(\frac{\mu}{r}-\frac{G \mu^{2}}{r^{2}}\right)+O\left(r^{-3}\right) .
$$

The effect of this on the geometry can be seen making $r^{2}=\rho^{2}+G^{2} \mu^{2}$ in (2.6) so that the $g_{\rho \rho}$ component of the metric, which behaves as

$$
g_{\rho \rho}=\frac{l^{2}}{\rho^{2}}+\left(1-\frac{3 G^{2} \mu^{2}}{l^{2}}\right) \frac{l^{4}}{\rho^{4}}+O\left(\rho^{-5}\right)
$$

is the only one relaxed in comparison with the bare black hole, for which

$$
g_{\rho \rho}^{0}=\frac{l^{2}}{\rho^{2}}+\frac{l^{4}}{\rho^{4}}+O\left(\rho^{-5}\right)
$$

In spite of the fact that the scalar field is more spread out and the geometry exhibits a greater deviation from AdS in the asymptotic region, the conserved charges are still well defined and finite.

\section{Acknowledgments}

We thank E. Ayón-Beato, G. Barnich, G. Compère and A. Gomberoff for useful discussions and enlightening comments. C. M. and R. T. wish to thank Prof. M. Henneaux for his kind hospitality at the Université Libre de Bruxelles. This research is partially funded by FONDECYT grants 1010446, 1010449, 1010450, 1020629, 1040921, 7010446, 7010450, and 7020629. The generous support to Centro de Estudios Científicos (CECS) by Empresas CMPC is also acknowledged. CECS is a Millennium Science Institute and is funded in part by grants from Fundación Andes and the Tinker Foundation. 


\section{A. Appendix}

The form of the self-interacting potential considered here (2.2) can be naturally obtained through the relation between the conformal and Einstein frames. Performing a conformal transformation, with a scalar field redefinition of the form

$$
\hat{g}_{\mu \nu}=\left(1-\frac{4 \pi G}{3} \Psi^{2}\right)^{-1} g_{\mu \nu}, \quad \Psi=\sqrt{\frac{3}{4 \pi G}} \tanh \sqrt{\frac{4 \pi G}{3}} \phi
$$

the action (2.1, 2.2) reads

$$
I\left[\hat{g}_{\mu \nu}, \Psi\right]=\int d^{4} x \sqrt{-\hat{g}}\left[\frac{\hat{R}+6 l^{-2}}{16 \pi G}-\frac{1}{2} \hat{g}^{\mu \nu} \partial_{\mu} \Psi \partial_{\nu} \Psi-\frac{1}{12} \hat{R} \Psi^{2}-\frac{2 \pi G}{3 l^{2}} \Psi^{4}\right] .
$$

In this frame, the matter action is invariant under arbitrary local rescalings $\hat{g}_{\mu \nu} \rightarrow \lambda^{2}(x) \hat{g}_{\mu \nu}$ and $\Psi \rightarrow \lambda^{-1} \Psi$, so that the scalar field equation is conformally invariant.

The black hole solution (2.6, 2.7) acquires a simple form once expressed in the conformal frame

$$
d \hat{s}^{2}=-\left(\frac{r^{2}}{l^{2}}-\left(1+\frac{G \mu}{r}\right)^{2}\right) d t^{2}+\left(\frac{r^{2}}{l^{2}}-\left(1+\frac{G \mu}{r}\right)^{2}\right)^{-1} d r^{2}+r^{2} d \sigma^{2}
$$

with

$$
\Psi=\sqrt{\frac{3}{4 \pi G}} \frac{G \mu}{r+G \mu} .
$$

Note that the mapping between both frames (A.1) is invertible in the region where the conformal factor $1-4 \pi G \Psi^{2} / 3$ is positive. That is, for nonnegative mass $(\mu \geq 0)$, for $r>0$, and for negative mass, $r>-2 G \mu$.

For nonnegative masses this solution possesses only one event horizon and it has the same causal structure as in the Einstein frame. However, for the rest of the allowed range, $-l / 4<$ $G \mu<0$, the metric develops three horizons, satisfying $0<r_{--}<-G \mu<r_{-}<l / 2<r_{+}$. From a cursory look, one may say that this solution seems to describe a black hole inside a black hole. In this frame, the scalar field becomes singular at $r=-G \mu$, but since the geometry as well as the stress-energy tensor are regular there, this singularity seems to be harmless. A detailed analysis of the causal and thermodynamic properties of the solution in the conformal frame is out of the scope of this appendix, and will be discussed elsewhere.

\section{References}

[1] R. Ruffini and J. Wheeler, Phys. Today 24(1), 30 (1971).

[2] J. D. Bekenstein, Phys. Rev. D 5, 1239 (1972). 
[3] D. Sudarsky, Class. Quant. Grav. 12, 579. (1995). A. Saa, J. Math. Phys. 37, 2346 (1996). A. E. Mayo and J. D. Bekenstein, Phys. Rev. D 54, 5059 (1996). A. Saa, Phys. Rev. D 53, 7377 (1996). J. D. Bekenstein, "Black hole hair: Twenty-five years after", arXiv:gr-qc/9605059. D. Sudarsky and T. Zannias, Phys. Rev. D58, 087502 (1998). K. A. Bronnikov and G. N. Shikin, Grav. Cosmol. 8, 107 (2002). D. Sudarsky and J. A. Gonzalez, Phys. Rev. D67, 024038 (2003). E. Ayón-Beato, Class. Quant. Grav. 19, 5465 (2002). U. Nucamendi and M. Salgado, Phys. Rev. D68, 044026 (2003).

[4] C. Martínez and J. Zanelli, Phys. Rev. D54, 3830 (1996).

[5] M. Henneaux, C. Martínez, R. Troncoso and J. Zanelli, Phys. Rev. D65, 104007 (2002).

[6] C. Martínez, R. Troncoso and J. Zanelli, Phys. Rev. D67, 024008 (2003).

[7] N. Bocharova, K. Bronnikov and V. Melnikov, Vestn. Mosk. Univ. Fiz. Astron. 6, 706 (1970). J. D. Bekenstein, Annals Phys. 82, 535 (1974); Annals Phys. 91, 75 (1975).

[8] T. Torii, K. Maeda and M. Narita, Phys. Rev. D64, 044007 (2001).

[9] E. Winstanley, Found. Phys. 33, 111 (2003).

[10] T. Hertog and K. Maeda, "Black holes with scalar hair and asymptotics in $N=8$ supergravity", arXiv:hep-th/0404261.

[11] J. P. S. Lemos, Phys. Lett. B352, 46 (1995).

[12] L. Vanzo, Phys. Rev. D56, 6475 (1997).

[13] D. R. Brill, J. Louko, and P. Peldan, Phys. Rev. D56, 3600 (1997).

[14] D. Birmingham, Phys.Rev. D59, 044013 (1999).

[15] R. G. Cai and K. S. Soh, Phys. Rev. D59, 044013 (1999).

[16] J. Crisóstomo, R. Troncoso and J. Zanelli, Phys. Rev. D62, 084013 (2000).

[17] R. Aros, R. Troncoso and J. Zanelli, Phys. Rev. D63, 084015 (2001).

[18] R. G. Cai, Phys. Rev. D65, 084014 (2002).

[19] M. H. Dehghani, "Asymptotically (anti)-de Sitter solutions in Gauss-Bonnet gravity without a cosmological constant", arXiv:hep-th/0405206.

[20] P. Breitenlohner and D. Z. Freedman, Phys. Lett. B115, 197 (1982); Annals Phys. 144, 249 (1982).

[21] L. Mezincescu and P. K. Townsend, Annals Phys. 160, 406 (1985).

[22] R. Aros, C. Martínez, R. Troncoso and J. Zanelli, JHEP 0205, 020 (2002).

[23] G. Gibbons and S. A. Hartnoll, Phys. Rev. D66, 064024 (2002).

[24] G. W. Gibbons and S. W. Hawking, Phys. Rev. D15, 2752 (1977).

[25] T. Regge and C. Teitelboim, Annals Phys. 88, 286 (1974).

[26] M. Henneaux and C. Teitelboim, Commun. Math. Phys. 98, 391 (1985).

[27] M. Henneaux, C. Martínez, R. Troncoso and J. Zanelli, "Asymptotically anti-de Sitter spacetimes and scalar fields with a logarithmic branch", arXiv:hep-th/0404236. 
[28] J. Gegenberg, C. Martínez and R. Troncoso, Phys. Rev. D67, 084007 (2003).

[29] G. Barnich and F. Brandt, Nucl. Phys. B633, 3 (2002). G. Barnich, "Conserved charges in gravitational theories: Contribution from scalar fields", arXiv:gr-qc/0211031. G. Barnich, Class. Quant. Grav. 20, 3685 (2003).

[30] E. Witten, Adv. Theor. Math. Phys. 2, 505 (1998).

[31] R. Aros, C. Martínez, R. Troncoso and J. Zanelli, Phys. Rev. D 67, 044014 (2003).

[32] M. Bianchi, D. Z. Freedman and K. Skenderis, JHEP 0108, 041 (2001). 